\title{
Hydrogen Embrittlement of Aluminum: The Crucial Role of Vacancies
}

\section{Citation}

Lu, Gang, and Efthimios Kaxiras. 2005. "Hydrogen Embrittlement of Aluminum: The Crucial Role of Vacancies." Physical Review Letters 94 (15). https://doi.org/10.1103/physrevlett.94.155501.

\section{Permanent link}

http://nrs.harvard.edu/urn-3:HUL.InstRepos:41384115

\section{Terms of Use}

This article was downloaded from Harvard University's DASH repository, and is made available under the terms and conditions applicable to Other Posted Material, as set forth at http:// nrs.harvard.edu/urn-3:HUL.InstRepos:dash.current.terms-of-use\#LAA

\section{Share Your Story}

The Harvard community has made this article openly available.

Please share how this access benefits you. Submit a story.

\section{Accessibility}




\title{
Hydrogen Embrittlement of Aluminum: the Crucial Role of Vacancies
}

\author{
Gang $\mathrm{Lu}^{(1)}$ and Efthimios Kaxiras ${ }^{(2)}$ \\ (1) Department of Physics, California State University Northridge, Northridge, California 91330 \\ ${ }^{(2)}$ Department of Physics and Division of Engineering and Applied Sciences, \\ Harvard University, Cambridge, Massachusetts 02138
}

\begin{abstract}
We report first-principles calculations which demonstrate that vacancies can combine with hydrogen impurities in bulk aluminum and play a crucial role in the embrittlement of this prototypical ductile solid. Our studies of hydrogen-induced vacancy superabundant formation and vacancy clusterization in aluminum lead to the conclusion that a large number of $\mathrm{H}$ atoms (up to twelve) can be trapped at a single vacancy, which over-compensates the energy cost to form the defect. In the presence of trapped $\mathrm{H}$ atoms, three nearest-neighbor single vacancies which normally would repel each other, aggregate to form a trivacancy on the slip plane of $\mathrm{Al}$, acting as embryos for microvoids and cracks and resulting in ductile rupture along the these planes.
\end{abstract}

Hydrogen degradation of the structural properties of solids, referred to as embrittlement, is a fundamental problem in materials physics. Despite intense studies, the definitive mechanism of $\mathrm{H}$ embrittlement in metals remains poorly understood. Four general mechanisms have been proposed: (i) formation of a hydride phase; (ii) enhanced local plasticity; (iii) grain boundary weakening and (iv) blister and bubble formation [1]. The underlying atomic processes and relative importance of the four mechanisms remain uncertain, and it is likely that a combination of these processes may contribute to embrittlement simultaneously. For these mechanisms to be operational, however, a critical local concentration of $\mathrm{H}$ is required, either to form a hydride phase or to initiate cracking at microvoids and grain boundaries. One of the outstanding problems in the current theories of hydrogen embrittlement is the lack of a comprehensive and coherent atomistic mechanism to account for the critical $\mathrm{H}$ concentrations at crack tips. Moreover, it is widely observed that $\mathrm{H}$-enhanced dislocation mobility is a prelude to the embrittlement and that the fracture planes coincide with the slip plane of the material, which is not the typical situation [1]; how all these phenomena come about still remains a mystery. It is generally believed that dislocations are central to $\mathrm{H}$ embrittlement phenomena, and a large body of work has been dedicated to elucidate hydrogen-dislocation interaction and its consequences on embrittlement 1, 2]. Vacancies, being ubiquitously present in solids and having the ability to act as impurity traps, could play a central role in the embrittlement process, but detailed arguments about this role or estimates of its relative importance are totally lacking.

Recent experiments on H-metal systems offer clues on the role that vacancies may play in $\mathrm{H}$ embrittlement. One set of experiments has established that $\mathrm{H}$ could induce superabundant vacancy formation in a number of metals, such as $\mathrm{Pd}, \mathrm{Ni}, \mathrm{Cr}$, etc. [3, 4]. The estimated vacancy concentration, $\mathrm{C}_{V}$, in these systems can reach a value as high as 23 at.\% 3]. A conclusion drawn from these experiments is that $\mathrm{H}$ atoms, originally at interstitial positions in the bulk, are trapped at vacancies in multiple numbers with rather high binding energies. It was speculated that several (three to six) $\mathrm{H}$ atoms can be trapped by a single vacancy, with the highest number (six) corresponding to the number of octahedral sites around a vacancy in either the fcc or the bcc lattice [3]. Actually, we shall show below based on first-principles theoretical calculations that in $\mathrm{Al}$, the prototypical simple metal and ductile solid, up to twelve $\mathrm{H}$ atoms can be trapped at a single vacancy site. The consequence of $\mathrm{H}$ trapping is that the formation energy of a vacancy defect is lowered by a significant amount, an energy that we define as the $\mathrm{H}$ trapping energy. Such reduction in the vacancy formation energy could result in drastic increase $\left(10^{7}\right.$ fold for $\mathrm{Fe}$ ) of equilibrium vacancy concentrations [5]. The superabundant vacancy formation in turn provides more trapping sites for $\mathrm{H}$ impurities, effectively increasing the apparent $\mathrm{H}$ solubility in metals by many orders of magnitude. For example, it was observed experimentally that about 1000 atomic parts per million (appm) of $\mathrm{H}$ atoms can enter $\mathrm{Al}$ accompanied by vacancy formation at the surface under aggressive $\mathrm{H}$ charging conditions, which should be contrasted with the equilibrium solubility of $\mathrm{H}$ in $\mathrm{Al}$ of about $10^{-5}$ appm at room temperature where the experiments were carried out $[\underline{6}]$; this is a staggering change of eight orders of magnitude in concentration. It was futher observed that the H-vacancy defects clustered and formed platelets lying on the $\{111\}$ planes, which directly lead to void formation or crack nucleation on the $\{111\}$ cleavage planes [6].

In order to elucidate the complex nature of H-vacancy interaction and to shed light on experimental results, we have performed first-principles calculations to examine the energetics and electronic structure for the relevant $\mathrm{H}$-vacancy complexes in Al. Due to the extremely low solubility of $\mathrm{H}$ in bulk $\mathrm{Al}$, experiments are usually difficult and results are dependent on $\mathrm{H}$ charging conditions; for such systems, first-principles calculations are particularly useful to complement experimental approaches. Our first-principles calculations are based on density functional theory with the VASP implementation [7] and ultra-soft pseudopotentials [8]. The local-density 


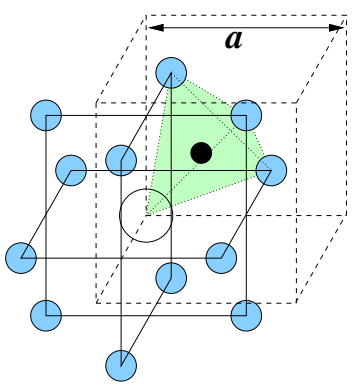

(a)

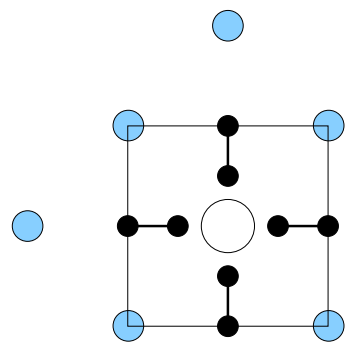

(b)
FIG. 1: Schematic representation of the environment of a vacncy in Al. (a) The vacancy as a large open circle and its 12 nearest neighbors as smaller grey circles, which lie on highlighted [100] planes. The cube in dashed lines represents the conventional cell of the FCC lattice of side $a$. A shaded tetrahedron with one corner at the vacancy site is also shown, and the geometric center of which corresponds to the lowestenergy site for a $\mathrm{H}$ interstitial atom (shown as black circle) in bulk Al. (b) The arrangment of four of the six $\mathrm{H}_{2}$ molecules surrounding the vacancy, on a [100] plane, with the first- and second-nearest-neighbor $\mathrm{Al}$ atoms indicated. The other two molecules lie directly above and below the plane of the figure, along an axis perpendicular to this plane passing throught the vacancy site. In both (a) and (b) the ions are placed at the ideal lattice sites, with the atomic relaxations not shown explicitly.

approximation (LDA) is used in all of our calculations, with checks based on the generalized gradient approximation (GGA) for selected cases. For Al, we find that LDA results are consistently closer to experimental values than GGA results, so here we will rely mainly on LDA numbers to draw physical conclusions. We employ a supercell containing 108 atomic sites in a simple cubic lattice to model bulk $\mathrm{Al}$, with a $4 \times 4 \times 4$ reciprocal space grid in the suprecell Brillouin zone and a plane-wave kinetic energy cutoff of $220 \mathrm{eV}$ for the Al-H system. With these parameters, we obtain the formation energy of a single vacancy $(0.66 \mathrm{eV})$ and the binding energy for the nearest-neighbour $(\mathrm{NN})$ divacancy in pure $\mathrm{Al}(-0.06 \mathrm{eV})$ in excellent agreement with other theoretical [9] and experimental results [10] (Table I). We note that the NN divacancy formation energy is negative, implying that it is unstable compared to two isolated single vacancies. This counter-intuitive result is due to charge redistribution in the neighborhood of the vacancy, which has been interpreted as formation of directional covalent/metallic bonds that stabilizes the single vacancy configuration against the formation of the divacancy [9, 11].

Our main objective is to understand the atomistic mechanisms of H-vacancy interaction in Al. First we address the relative site preference of $\mathrm{H}$ in bulk $\mathrm{Al}$. To this end, we have calculated the total energy of a single $\mathrm{H}$ atom situated near the vacancy site, or at intersti- tial tetrahedral and octahedral bulk sites which are as far as possible from the vacancy within the supercell. For $\mathrm{H}$ atoms, the tetrahedral interstitial site in bulk $\mathrm{Al}$ is slightly more favorable than the octahedral interstitial site by $0.07 \mathrm{eV}$. We find that the $\mathrm{H}$ atom prefers to occupy the vacancy site over the interstitial tetrahedral site in bulk by $0.40 \mathrm{eV}$. The corresponding experimental value is $0.52 \mathrm{eV}$ [1], and theoretical results range from $0.33 \mathrm{eV}$ to about $1 \mathrm{eV}[12,13$. The lowest energy position for the $\mathrm{H}$ atom in the presence of a vacancy is not at the geometric center of the vacancy site, but rather at an off-center position close to a tetrahedral site adjacent to the vacancy site (see Fig. 1(a)); the energy difference between the center and off-center positions is 0.66 $\mathrm{eV}$. We also find that the $\mathrm{H}$ atom is negatively charged, consistent with the view that the $\mathrm{H}$ impurity can be regarded as a screened $\mathrm{H}^{-}$ion in free-electron-like metals 14]. Previous studies based on the jellium model of $\mathrm{Al}$ have shown that as the jellium conduction electron density decreases, the excess charge buildup at the $\mathrm{H}$ atom is also reduced and the electrons of the $\mathrm{H}^{-}$ion are less localized [14, 15]. Therefore, the kinetic energy of the $\mathrm{H}^{-}$ electrons is lowered at the vacancy site where the conduction electron density is lower. At the same time, it is energetically favorable for the $\mathrm{H}^{-}$ion to sit off-center of the vacancy, to minimize the Coulomb interaction energy with the nearby $\mathrm{Al}$ ions.

Having established the stability of a single $\mathrm{H}$ atom at a single vacancy in $\mathrm{Al}$, the ensuing question is whether multiple $\mathrm{H}$ atoms, in particular, $\mathrm{H}_{2}$ molecules would be stable at this defect. This is an interesting problem on its own right, but it is also relevant to $\mathrm{H}_{2}$ bubble formation that gives rise to $\mathrm{H}$ embrittlement. To examine the stability of an $\mathrm{H}_{2}$ molecule at a vacancy site, we compare the binding energy of the $\mathrm{H}_{2}$ unit at the vacancy and in vacuum. The binding energy $E_{b}$ of the $\mathrm{H}_{2}$ unit at a vacancy site is calculated as:

$$
E_{b}=E_{c}\left(V_{\mathrm{Al}}+\mathrm{H}_{2}\right)+E_{c}\left(V_{\mathrm{Al}}\right)-2 E_{c}\left(V_{\mathrm{Al}} \mathrm{H}\right),
$$

where $E_{c}\left(V_{\mathrm{Al}}+\mathrm{H}_{2}\right)$ is the cohesive energy of a system with an $\mathrm{H}_{2}$ unit at the center of the vacancy, $E_{c}\left(V_{\mathrm{Al}}\right)$ is the cohesive energy of a system with a single vacancy in the absence of the $\mathrm{H}_{2}$ unit, and $E_{c}\left(V_{\mathrm{Al}} \mathrm{H}\right)$ is the cohesive energy of a system with a single $\mathrm{H}$ atom at the vacancy (in the off-center tetrahedral site). Interestingly, we find the this binding energy to be $+0.06 \mathrm{eV}$, indicating a weak repulsion between the two $\mathrm{H}$ atoms in the $\mathrm{H}_{2}$ unit at the vacancy site. This is to be compared to the binding energy of an $\mathrm{H}_{2}$ molecule in vacuum, which is $-6.67 \mathrm{eV}$. The positive binding energy of $\mathrm{H}_{2}$ at the vacancy site does not imply that there is no bonding between the two $\mathrm{H}$ atoms; it simply states that these two $\mathrm{H}$ atoms would prefer to be trapped at two single vacancy sites individually rather than in the same vacancy site as a pair. The weakening of the $\mathrm{H}-\mathrm{H}$ bond at the vacancy site is remarkable given the fact that each $\mathrm{H}$ atom in the $\mathrm{H}_{2}$ unit in 


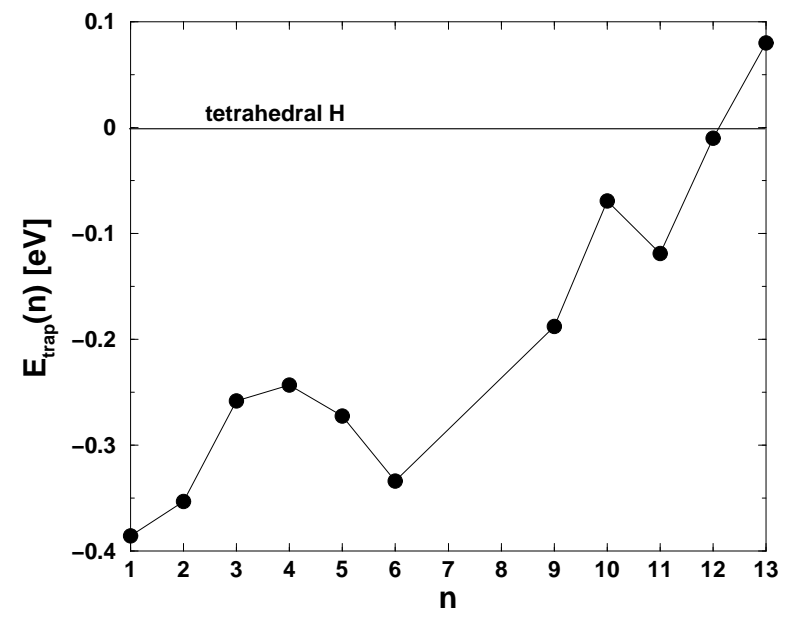

FIG. 2: Trapping energy per $\mathrm{H}$ atom in $\mathrm{eV}$ as a function of the number of $\mathrm{H}$ atoms being trapped at a single vacancy site. The zero energy corresponds to the energy of a $\mathrm{H}$ atom at the tetrahedral interstitial site.

this situation is quite far away $(2.6 \AA)$ from the nearest $\mathrm{Al}$ ions.

We find that the equilibrium interatomic distance between the $\mathrm{H}$ atoms at the vacancy is $0.83 \AA$, $12 \%$ longer than the $\mathrm{H}_{2}$ bond length $(0.74 \AA)$ of the molecule in vacuum; this is due to the partial occupation of antibonding states between the $\mathrm{H}$ atoms. This can be understood as follows: each $\mathrm{H}$ atom is associated with a doubly occupied bound state in the presence of conduction electrons, and hence is negatively charged. When the two $\mathrm{H}$ atoms approach each other, the two bound states split up into a bonding and an antibonding level. In contrast to what happens in vacuum, the screening of the conduction electrons reduces the bonding-antibonding energy splitting, and the antibonding level may be occupied by conduction electrons if the Fermi energy of the metal is high enough 15. The occupation of antibonding states weakens the $\mathrm{H}_{2}$ bond and increases the bond length. Our results agree qualitatively with the jellium model calculations which also found the $\mathrm{H}_{2}$ binding energy to be positive and the bond length increased, ranging from 0.81 to $0.86 \AA$ depending on the jellium density. In particular, for low jellium electron density (corresponding to the center of a vacancy site in $\mathrm{Al}$ ), the binding energy was found to be $+0.02 \mathrm{eV}[16$. Similarly, one can calculate the binding energy of multiple $\mathrm{H}$ atoms trapped at a single vacancy site, which turn out to be positive as well. Based on these results, we conclude that if the single vacancy concentration $\mathrm{C}_{V}$ is greater than the $\mathrm{H}$ concentration $\mathrm{C}_{H}$, each vacancy in equilibrium should contain no more than one $\mathrm{H}$ atom.

On the other hand, if $\mathrm{C}_{H}$ is greater than $\mathrm{C}_{V}$, the question arises as to where will the extra $\mathrm{H}$ atoms be situated, at interstitial or at vacancy sites? Experimental measurements for the ratio $\mathrm{C}_{H} / \mathrm{C}_{V}$ in $\mathrm{Al}$ range from 0.25 to 4 , depending on $\mathrm{H}$ charging conditions, with the most probable value close to 1 [ $]$. To answer the above question, we have calculated the trapping energy $E_{\text {trap }}$ of multiple $\mathrm{H}$ atoms at a single vacancy site, which is defined as:

$$
E_{\text {trap }}(n)=\frac{1}{n}\left[E_{c}\left(V_{\mathrm{Al}}+n \mathrm{H}\right)-E_{c}\left(V_{\mathrm{Al}}\right)\right]-\left[E_{c}^{0}(\mathrm{H})-E_{c}^{0}\right],
$$

where $E_{c}\left(V_{\mathrm{Al}}+n \mathrm{H}\right)$ is the cohesive energy of a system with $n \mathrm{H}$ atoms each situated at a single vacancy site, $E_{c}^{0}(\mathrm{H})$ is the cohesive energy of bulk $\mathrm{Al}$ with a $\mathrm{H}$ atom at the tetrahedral interstitial site, and $E_{c}^{0}$ is the cohesive energy of the ideal bulk without $\mathrm{H}$. A negative value for the trapping energy represents the energy gain when the $\mathrm{H}$ atoms are trapped at a single vacancy site relative to being dispersed at $n$ different tetrahedral interstitial sites. The results for $E_{\text {trap }}$ as a function of $n$ are summarized in Fig. 2. Consistent with the binding energy calculations, it is energetically most favorable for each vacancy to trap a single $H$ atom. At the same time, it is also energetically favorable for multiple $\mathrm{H}$ atoms to be trapped at a single vacancy site relative to being dispersed at interstitial sites as individual atoms. In fact, up to twelve $\mathrm{H}$ atoms can be trapped at a single vacancy in $\mathrm{Al}$, twice the highest number of $\mathrm{H}$ atoms (six) that can be trapped in $\mathrm{Fe}$ [5]. The atomic arrangement of the 12 $\mathrm{H}$ atoms trapped at a single vacancy is indicated in Fig. 1(b). There are two $\mathrm{H}_{2}$ units in each $\langle 100\rangle$ direction surrounding the vacancy, and the bond length is $1 \AA$ for all six units. The inter-molecule distance in each direction is $3 \AA$, and the NN distance between $\mathrm{H}$ and $\mathrm{Al}$ in each direction is $2 \AA$ (the lattice constant of $\mathrm{Al}$ is $3.99 \AA$ ). The ordered arrangement of the $\mathrm{H}$ atoms is necessary to minimize the electrostatic energy. The greater H-trapping capacity of $\mathrm{Al}$ compared to $\mathrm{Fe}$, can be attributed to its larger lattice constant and more delocalized nature of electrons. It is observed that the volume change of the supercell owing to the $\mathrm{H}$ additions is negligible.

The fact that the $\mathrm{H}_{2}$ units at a single vacancy site attract the conduction electrons from the edge of the vacancy, raises the interesting possibility that the covalent/metallic bonds between the first shell of $\mathrm{NN} \mathrm{Al}$ ions around the vacancy site may be disrupted enough to permit a coalescence of multiple vacancies. To check this possibility, we carried out calculations for a number of relevant configurations. Specifically, we have examined: (i) two vacancies, each with one $\mathrm{H}$ atom, forming a $\mathrm{NN}$ divacancy with two $\mathrm{H}$ atoms trapped; (ii) $n$ vacancies, each with two $\mathrm{H}$ atoms, forming a complex of NN multivacancies with $2 n \mathrm{H}$ atoms trapped, for $n=2$ and 3 . To summarize the results, we use the notation of chemical reactions:

$$
\begin{aligned}
2 V_{\mathrm{Al}} \mathrm{H} & \rightarrow\left(V_{\mathrm{Al}}\right)_{2} \mathrm{H}_{2}-0.21 \mathrm{eV} \quad(\mathrm{i}) \\
n V_{\mathrm{Al}} \mathrm{H}_{2} & \rightarrow\left(V_{\mathrm{Al}}\right)_{n} \mathrm{H}_{2 n}+n 0.29 \mathrm{eV}
\end{aligned}
$$

where the last number in each equation represents the reaction enthalpy $\Delta H$. A positive value of $\Delta H$ means the 
reaction is exothermic, that is, the process from left to right is energetically favorable. $\Delta H$ is defined as follows for reaction (i):

$$
\Delta H=2 E_{c}\left(V_{\mathrm{Al}} \mathrm{H}\right)-E_{c}\left[\left(V_{\mathrm{Al}}\right)_{2} \mathrm{H}_{2}\right]-E_{c}^{0},
$$

where $E_{c}\left[\left(V_{\mathrm{Al}}\right)_{2} \mathrm{H}_{2}\right]$ is the cohesive energy of a system with two $\mathrm{H}$ atoms trapped at a divacancy, with analogous definitions for reaction (ii). Consistent with our earlier discussion, we find reaction (i) to be unfavorable (endothermic) because the effect of a single $\mathrm{H}$ atom on the covalent/metallic bonding of the $\mathrm{NN} \mathrm{Al}$ atoms around the vacancy site is small and localized. On the other hand, reaction (ii) is favorable for $n=2$ and 3 , because the $\mathrm{H}_{2}$ units can attract more conduction electrons from the nearby $\mathrm{Al}$ atoms, weakening the bonding among the NN $\mathrm{Al}$ atoms, which in turn drives the formation of multivacancies. The large energy gain in forming the trivacancy $(n=3)$ is of particular interest. First, it is consistent with the experimental observation that the single vacancy defects occupied by $\mathrm{H}$ atoms can coalescence to form platelets on $\{111\}$ planes of Al. Although our calculations primarily concern the formation of the trivacancy, it is likely that even larger vacancy clusters can also be formed based on the same mechanism. In support of this claim, we mention that the increase in positive enthalpy associated with reaction (ii) is linear in the number of vacancies for $n=2$ and 3. Second, these vacancy clusters can serve as embryos of cracks and microvoids with local $\mathrm{H}$ concentrations much higher than the average bulk value.

Next we discuss the implications of our results on hydrogen embrittlement phenomena. It was generally believed that $\mathrm{H}$-induced embrittlement in metals takes the form of plastic rupture rather than brittle fracture, consistent with the notion of $\mathrm{H}$-enhanced local plasticity (HELP). It was widely observed that the fracture surface is along the active slip planes where shear localization occurs. For fcc metals, the slip planes are the $\{111\}$ planes. In many cases, microvoids open up along these active slip planes in front of the crack tip; these microvoids can open and close in response to the local stress. Plastic rupture occurs when these microcracks are joined to the crack tip, upon reaching the critical stress. Our results clearly suggest that $\mathrm{H}$-enriched microvoids may be created along the slip planes by the coalescence of vacancies with trapped H. These microvoids can be formed only in the presence of $\mathrm{H}$, which produces an additional source of microcracks necessary for the $\mathrm{H}$ embrittlement. Moreover, the H-induced vacancy formation also facilitates dislocation climb, leaving behind vacancy rows in the highly deformed regions, which may contribute to the formation of microcracks as well. Our studies, taken together with the observed vacancy-enhanced dislocation glide [17, 18], suggest that vacancies are also responsible for the HELP phenomena that are a prelude to $\mathrm{H}$ embrittlement [1]. The fact that there is a strong binding between $\mathrm{H}$ and dislocation cores, and $\mathrm{H}$ can enhance dislocation motion along the slip planes 19], provide a means of rapid transport of $\mathrm{H}$ atoms to the crack front. On the other hand, the apparent lattice mobility of $\mathrm{H}$ atoms is also enhanced since multiple $\mathrm{H}$ atoms may be trapped at a single vacancy. All these vacancy-based mechanisms contribute to the $\mathrm{H}$ embrittlement as they increase the rate of crack growth. Finally, the significant $\mathrm{H}$ trapping at vacancies provides a scenario by which drastic increase of local $\mathrm{H}$ concentration may occur without improbable accumulation of $\mathrm{H}$ at bulk interstitial sites [5]. This new feature resolves the long-standing problem of how a sufficiently high $\mathrm{H}$ concentration can be realized to successfully induce $\mathrm{H}$ embrittlement in materials such as $\mathrm{Al}$, where the equilibrium $\mathrm{H}$ concentration in bulk is extremely low.

We acknowledge the support from Grant No. F4962099-1-0272 through the U.S. Air Force Office for Scientific Research.

[1] S. M. Myers et al, Rev. Mod. Phys. 64, 559 (1992) and reference therein.

[2] H. K. Birnbaum, in Hydrogen Embrittlement and Stress Corrosion Cracking, edited by R. Gibala and R.F. Hehemann (Metals Park, Ohio, 1984).

[3] Y. Fukai, Phys. Scripta, T103, 11 (2003).

[4] Y. Fukai and N. Ōkuma, Phys. Rev. Lett. 73, 1640 (1994).

[5] Y. Tateyama and T. Ohno, Phys. Rev. B 67, 174105 (2003).

[6] H. K. Birnbaum et al., J. Alloys Comp. 253-254, 260 (1997).

[7] G. Kresse and J. Furthmüller, Phys. Rev. B 54, 11169 (1996).

[8] D. Vanderbilt, Phys. Rev. B 41, 7892 (1990).

[9] K. Carling and G. Wahnström, Phys. Rev. Lett. 85, 3862 (2000).

[10] P. Ehrhart et al., in Atomic Defects in Metal, LandoltBörnstein, New Series, Group III, Vol. 25 (SpringerVerlag, Berlin, 1991).

[11] T. Uesugi, M. Kohyama, and K. Higashi, Phys. Rev. B 68, 184103 (2003).

[12] A. De Vita and M. J. Gillan, J. Phys.: Condens. Matter 4, 599 (1992).

[13] C. Wolverton, V. Ozolins, and M. Asta, Phys. Rev. B 69, 144109 (2004).

[14] J. K. Norskov, Phys. Rev. B 20, 446 (1979).

[15] J. K. Norskov, Solid State Commun. 24, 691 (1977), ibid. 25, 995 (1978).

[16] S. A. Bonev and N. M. Ashcroft, Phys. Rev. B 64, 224112 (2001).

[17] J. Lauzier, J. Hillairet, A. Vieux-Champagne, and W. Benoit, J. Phys. Condens. Matter, 1, 9273 (1989); J. Lauzier, J. Hillairet, G. Gremaud and W. Benoit, ibid, 2, 9247 (1990).

[18] W. Benoit, G. Gremaud and B. Quenet, Mater. Sci. Eng. A 164, 42 (1993).

[19] G. Lu, Q. Zhang, N. Kioussis, and E. Kaxiras, Phys. Rev. Lett. 87, 095501 (2001). 
TABLE I: The vacancy formation energy, $\Delta H_{V}^{F}$; the binding energy for the divacancy, $\Delta H_{2 V}^{b}=2 \Delta H_{V}^{F}-\Delta H_{2 V}^{F}$, where the last term is the formation energy of the divacancy. The total energy with a $\mathrm{H}$ atom occupying the octahedral interstitial site is set to zero, relative to which the total energy of a $\mathrm{H}$ atom occupying the tetrahedral interstitial site, $\mathrm{E}_{T}$, and the total energy of a $\mathrm{H}$ atom trapped at a single vacancy, $\mathrm{E}_{V}$ are defined. The last two columns are LDA and GGA results from other theoretical calculations. All energies are given in $\mathrm{eV}$. The experimental values marked by an asterisk have been called into question due to incorrect interpretations on the experimental part, see ref. [9] for details.

\begin{tabular}{cccccc}
\hline \hline & LDA & GGA & Exp.[10] & LDA & GGA \\
\hline$\Delta H_{V}^{F}$ & 0.66 & 0.54 & $0.67 \pm 0.03$ & $0.70[9]$ & $0.54[9]$ \\
$\Delta H_{2 V}^{b}$ & -0.06 & -0.07 & $0.2^{*}, 0.3^{*}$ & $-0.07[9]$ & $-0.08[9]$ \\
$\mathrm{E}_{T}$ & -0.07 & & & $-0.05[13]$ & $-0.13[13]$ \\
$\mathrm{E}_{V}$ & -0.47 & & & & $-0.46[13]$ \\
\hline \hline
\end{tabular}

[20] Using the energy curve between equilibrium and metastable positions of $\mathrm{H}$ in bulk $\mathrm{Al}$, we estimated the tunneling probability between equivalent equilibrium positions to be $3 \times 10^{-8}$, and thus quantum effects are not expected to be significant. Thermal effects (the configurational and vibrational entropy) are well beyond the reach of first-principles calculations for this system. 